\title{
Small Antiferromagnetic Spin Systems: Just beyond the Rotational Band Model
}

\author{
W. FLOREK ${ }^{a, *}$ AND L.A. KALISZAN ${ }^{b}$ \\ ${ }^{a}$ Adam Mickiewicz University, Faculty of Physics, Umultowska 85, 61-614 Poznań, Poland \\ ${ }^{b}$ Poznań School of Logistics, Estkowskiego 6, 61-755 Poznań, Poland
}

Two systems of spins $s=1 / 2$ with the Heisenberg interactions are investigated: (i) an equilateral trapezoid and (ii) a regular hexagon. Both cases are compared with the corresponding sublattice Hamiltonians to determine splitting and mixing of energy levels with a given total spin of sublattices. It is shown that small modifications of the Hamiltonian parameters may significantly change (magnetic) properties of the eigenstates, especially probability of finding system in a state with determined value of the sublattice total spin.

DOI: 10.12693/APhysPolA.127.330

PACS: 75.10.Jm, 75.50.Ee, 75.50.Xx

\section{Introduction}

The simplified picture of magnetic molecules is provided by the so-called rotational-band or sublattice model $[1,2]$ :

$$
\mathcal{H}_{\mathrm{SM}}=2 J \boldsymbol{S}_{A} \cdot \boldsymbol{S}_{B}=J\left[\boldsymbol{S}^{2}-\left(\boldsymbol{S}_{A}^{2}+\boldsymbol{S}_{B}^{2}\right)\right] \text {, }
$$

where $\boldsymbol{S}_{A(B)}$ is the total spin of sublattices $A$ and $B$, respectively, and $\boldsymbol{S}=\boldsymbol{S}_{A}+\boldsymbol{S}_{B}$ is the total spin of the system; $0 \leq S_{A(B)} \leq m s, 0 \leq S \leq 2 m s$, and the total magnetization $M$ are good quantum numbers. For antiferromagnetic (AFM) interactions the Hamiltonian (1) yields the singlet ground state (GS) with $S=0$,
$S_{A}=S_{B}=m s$. However, the AFM isotropic Heisenberg Hamiltonian with the nearest neighbors $(\mathrm{nnH})$ interactions leads to lower GS energy [3, 4]. Moreover, the eigenstates of these Hamiltonians are different. For the SM the GS with $S=M=0$ is the generalized Néel state [5, 6], where $S_{A}=S_{B}=m s$ and $-m s \leq M_{A(B)} \leq m s$. The actual GS of the nnH Hamiltonian is a linear combination of all states with $S=0$ (so $M=0$ ). The aim of our work is to consider eigenstates modifications in two simple cases with $s=1 / 2$; for such systems all calculations can be done exactly.
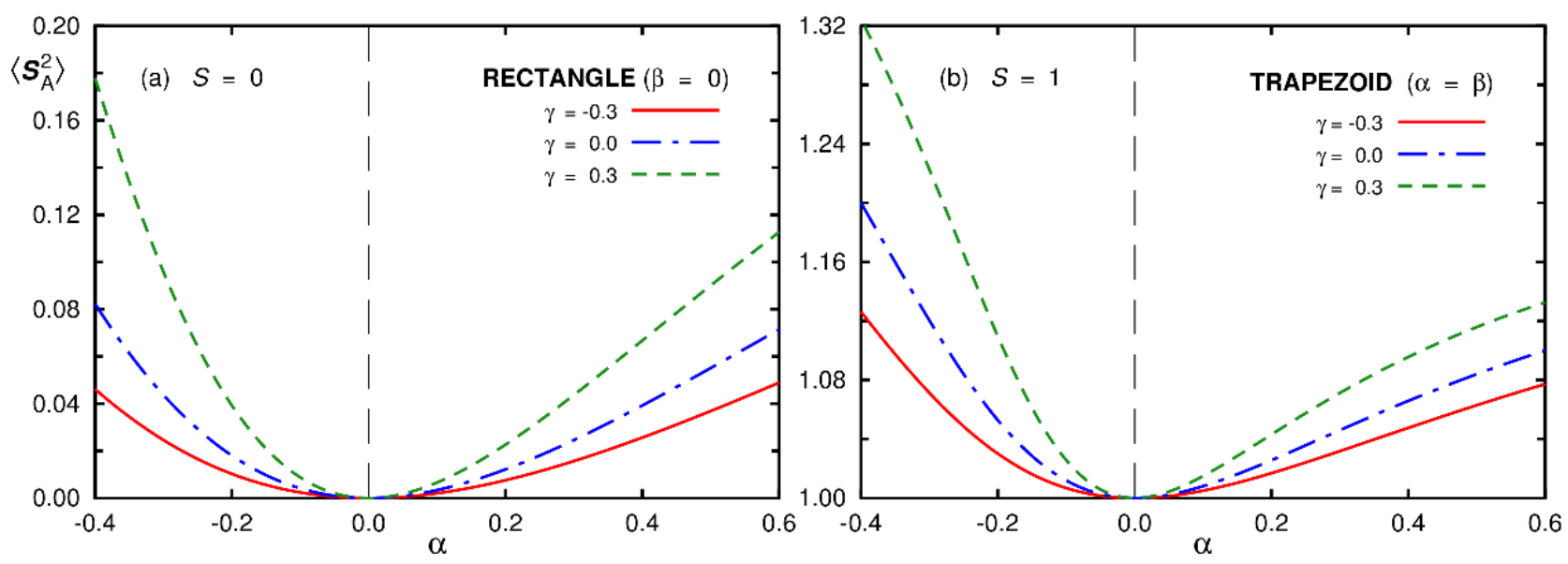

Fig. 1. $\left\langle\boldsymbol{S}_{A}^{2}\right\rangle$ in one of two states with (a) $S=0$ and (b) $S=1$.

\section{Geometrical deformations}

The AFM Hamiltonian is considered $(H=\mathcal{H} / J$, $J>0$ ), where small parameters $\alpha$ and $\beta$ describe deformation of a square $(\gamma \approx 0)$ or a tetrahedron $(\gamma \approx 1)$ to a trapezoid or a rectangle $(\beta=0)$ :

*Wojciech.Florek@amu.edu.pl

$$
\begin{array}{r}
H=2\left[\boldsymbol{s}_{2} \cdot \boldsymbol{s}_{3}+(1+\alpha)\left(\boldsymbol{s}_{1} \cdot \boldsymbol{s}_{2}+\boldsymbol{s}_{3} \cdot \boldsymbol{s}_{4}\right)\right. \\
\left.\quad+(1+2 \beta) \boldsymbol{s}_{1} \cdot \boldsymbol{s}_{4}+\gamma\left(\boldsymbol{s}_{1} \cdot \boldsymbol{s}_{3}+\boldsymbol{s}_{2} \cdot \boldsymbol{s}_{4}\right)\right]
\end{array}
$$

Since $S^{z}$ commutes with $\mathcal{H}$, it is enough to consider a sixdimensional space of states with $M=0$. To determine the mean values $\left\langle\boldsymbol{S}_{A(B)}^{2}\right\rangle$, the vectors $\left|S ; S_{A} S_{B}\right\rangle$, with $S=0,1,2$ and $S_{A(B)}=0,1$, are determined. The unique non-trivial symmetry $(1 \leftrightarrow 4,2 \leftrightarrow 3)$ gives us three symmetric states, $|2 ; 11\rangle,|0 ; 00\rangle,|0 ; 11\rangle$, and the only one 
antisymmetric state $|1 ; 11\rangle$. For the other two states combinations are considered

$$
|S=1\rangle_{ \pm}=2^{-1 / 2}(|1 ; 10\rangle \pm|1 ; 01\rangle) ;
$$

note that the first one is antisymmetric with respect to the trapezoid symmetry; in both states $\left\langle\boldsymbol{S}_{A(B)}^{2}\right\rangle=1$.

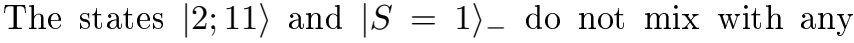
other state due to the symmetry. So we are left with two symmetric states for $S=0$ and two antisymmetric states for $S=1$. Two special cases are discussed below.

For $\beta=0$ the system can be considered as a rectangle. In this case only the symmetric states with $S=0$ and $S_{A}=S_{B}=0,1$ form linear combinations with $\left\langle\boldsymbol{S}_{A(B)}^{2}\right\rangle=\mathcal{S}$ and $2-\mathcal{S}$, respectively (see Fig. 1). Changes in the parameter $\gamma$ lead to quantitative only modification of the diagram, but $\mathcal{S}$ increases for growing $\gamma$. It can be shown that $\mathcal{S}=1$ for $\alpha=2(\gamma-1)$ when $\gamma \neq 1$. The dependence $\mathcal{S}(\alpha)$ is asymmetric: for $0<\gamma<1 \mathcal{S}(\alpha)$ grows more rapidly for $\alpha<0$ (it tends to $3 / 2$ ) than for $\alpha>0$ $\left(\lim _{\alpha \rightarrow \infty} \mathcal{S}(\alpha)=1 / 2\right)$.

If $\alpha=\beta$ then the mixing of $S_{A(B)}$-eigenstates is possible for antisymmetric states with $S=1$ only. The eigenstates have $\left\langle S_{A(B)}^{2}\right\rangle=1+\mathcal{S}$ and $2-\mathcal{S}$, respectively (see Fig. 1). Though this diagram is qualitatively similar to the previous one, the mixing of states is more intensive. It can be shown that $\lim _{\alpha \rightarrow \pm \infty} \mathcal{S}=(5 \mp \sqrt{5}) / 10$.

In a general case $(\alpha \neq \beta)$ the mixing of the eigenstates of the sublattice spins $S_{A(B)}^{2}$ is present for both values $S=0,1$. The results obtained are, at least qualitatively, similar to those presented above.

\section{A regular hexagon}

The system of six spins $s=1 / 2$ with the AFM Heisenberg coupling is considered $(0 \leq \alpha \leq 1)$ :

$$
H=\sum_{j=1}^{6} \boldsymbol{s}_{j} \cdot \boldsymbol{s}_{j+1}+\alpha \sum_{j=1}^{3} \boldsymbol{s}_{j} \cdot \boldsymbol{s}_{j+3} \text {. }
$$

For $\alpha=1$ the SM is revealed, whereas $\alpha=0$ leads to the nnH Hamiltonian. The coupling of the second neighbors does not yield any qualitative changes. The considerations are restricted to 20 states with $M=0$.

$S_{A}=S_{B}=3 / 2$ for $S=3$ and in one of states with $S=$ 2. The other four states with $S=2$ are (anti)symmetric combinations of states with $S_{A} \neq S_{B}$. The appropriate coefficients do not depend on $\alpha$ and the probability of $S_{A}=3 / 2$ is constant: $p=50 \%$. The same result is obtained for one pair of states with $S=1$.

Considering each of the operators $S_{A(B)}^{2}$ individually it is easy to notice that states with $S_{A(B)}=1 / 2$ (and fixed $M_{A}$ ) are related to two-dimensional representation $E^{\prime}$ of the dihedral group $\boldsymbol{D}_{3}$. Therefore, the level $|S ; 1 / 2,1 / 2\rangle, S=0,1$, is four-fold degenerated and splits to levels labeled by irreps $A_{1}^{\prime}, A_{2}^{\prime}$, and $E^{\prime}$ (the apostrophe distinguishes them from the irreps of $\left.\boldsymbol{D}_{6}\right)$. To obtain the eigenstates of (4) labeled by the irreps of $\boldsymbol{D}_{6}$, linear combinations with the fully symmetric state $|S ; 3 / 2,3 / 2\rangle$ (labeled by $A_{1}^{\prime}$ ) have to be introduced. For $S=1$ there is another level $E^{\prime}$ related to doubly degenerated levels $|1 ; 1 / 2,3 / 2\rangle$ and $|1 ; 3 / 2,1 / 2\rangle$, so the mixing of $\boldsymbol{S}_{A(B)^{-}}^{2}$ eigenstates is possible in this case, too. The other four states (labeled by $A_{2}^{\prime}$, and $E^{\prime}$ for $S=0$, and by $A_{2}^{\prime}$ for $S=1$ ) have determined values $S_{A(B)}=1 / 2$.

The linear combinations of the states labeled by $A_{1}^{\prime}$ $(S=0,1)$ have the symmetry $A_{1}$ for $S=1$ and $B_{1}$ for $S=0$ (one of these states is the GS of the Hamiltonian considered). The probabilities of measured given values of $S_{A(B)}$ are determined by the probability $p(\Gamma, \alpha)$ of $S_{A}=3 / 2$ in one state of each pair; in this state $\left\langle\boldsymbol{S}_{A(B)}^{2}\right\rangle=3 p+3 / 4$. These probabilities for $\Gamma=A_{1}$ and $B_{1}$ are presented in Fig. 2. In the nnH model we have $p\left(B_{1}, 0\right)=2 /(13+3 \sqrt{13}) \approx 8.4 \%$ and $p\left(A_{1}, 0\right)=2 /(9+3 \sqrt{5}) \approx 12.7 \%$.

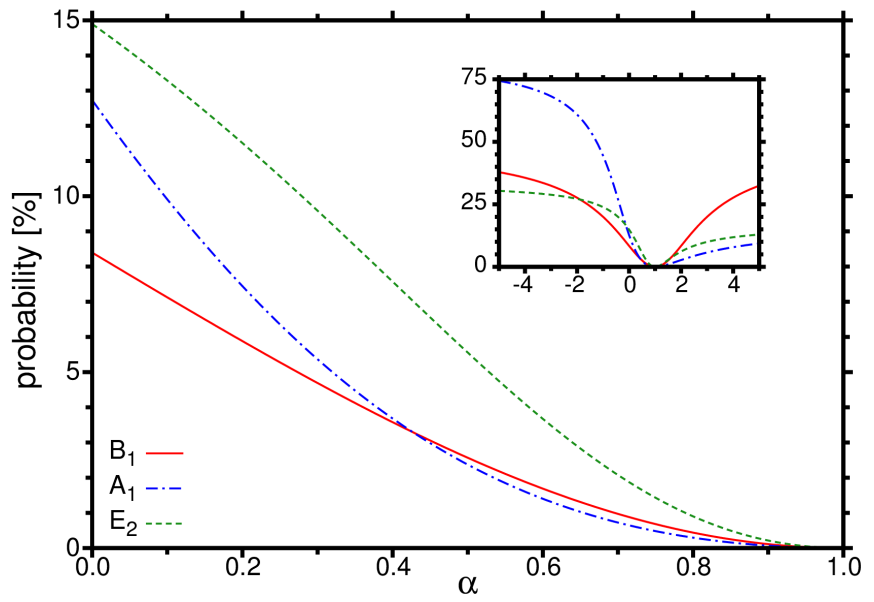

Fig. 2. The probabilities $p(\Gamma, \alpha)$ for $\Gamma=A_{1}, B_{1}, E_{2}$ and $0 \leq \alpha \leq 1$. In the inset $|\alpha| \leq 5$.

The doubly degenerated levels labeled by $E_{2}$ for $S=1$ are linear combinations of states labeled by $E^{\prime}:(|1 ; 3 / 2,1 / 2\rangle-|1 ; 1 / 2,3 / 2\rangle) / \sqrt{2}$ and $|1 ; 1 / 2,1 / 2\rangle$. The calculated probability $p\left(E_{2}, \alpha\right)$ (of $S_{A}=3 / 2$ ) is plotted in Fig. $2 ; p\left(E_{2}, 0\right)=32 / 3(51+5 \sqrt{17}) \approx 14.9 \%$.

\section{Final remarks}

The magnetic molecules synthesized never have ideal symmetry (for example see $[7,8]$ ), so considerations of spin systems with broken symmetry is important to better understand properties of real magnetic molecules. The examples discussed in this paper show that even small modifications in Heisenberg-like spin Hamiltonians may lead to distinct changes in properties of the corresponding eigenstates. On the contrary, small changes of Hamiltonian parameters do not influence the thermodynamic quantities, like the specific heat, since the statistical sum is slightly changed, especially at low temperatures. Therefore, fitting of microscopic parameters to experimental data based on the thermodynamic properties frequently leads to ambiguous results. Moreover, statedependent parameters, like spin-spin correlations, may 
be significantly different in states with similar energies (see, for example, some plots in [3]).

Similar effects are observed when a simplified model, like the sublattice one, is applied: the first energy gap, determining the specific heat at low temperatures, is well approximated by such models, but other properties of the eigenstates (especially excited ones) are not predicted properly.

\section{Acknowledgments}

The authors thank Prof. G. Kamieniarz for many helpful discussions. This work was supported in part by the National Science Center (Poland) within the project N202 230137.

\section{References}

[1] O. Waldmann, Phys. Rev. B 65, 024424 (2001).

[2] L. Engelhardt, M. Luban, Phys. Rev. B 73, 054430 (2006).

[3] W. Florek, S. Bucikiewicz, Phys. Rev. B 66, 024411 (2002).

[4] G. Kamieniarz, P. Kozłowski, G. Musiał, W. Florek, M. Antkowiak, M. Haglauer, A. Caramico D'Auria, F. Esposito, Inorg. Chim. Acta 361, 3690 (2008).

[5] P.L. Iske, W.J. Caspers, Physica 142A, 360 (1987).

[6] W. Florek, L.A. Kaliszan, H.W. Kunert, A.G.J. Machatine, Physica B 405, 3811 (2010).

[7] E.J. Schelter, A.V. Prosvirin, K.R. Dubnar, J. Am. Chem. Soc. 126, 15004 (2004).

[8] N. Hoshino, M. Nakano, H. Nojiri, W. Wernsdorfer, H. Oshio, J. Am. Chem. Soc. 131, 15100 (2009). 